\title{
Latina Mothers Recommendations on a Mindfulness-Based Cognitive Behavioral Therapy Group Intervention for Perinatal Depression
}

Kritzia Merced ( $\nabla$ k.mercedmorales@utah.edu )

University of Utah

Uma D. Parameswaran

University of Utah

Susan Dearden

University of Utah

Ryoko Pentecost

University of Utah

Gwen Latendresse

University of Utah

\section{Research Article}

Keywords: Latina women, perinatal depression, telehealth, mindfulness-based cognitive behavioral therapy, group therapy

Posted Date: November 18th, 2021

DOI: https://doi.org/10.21203/rs.3.rs-1028850/v1

License: (c) (1) This work is licensed under a Creative Commons Attribution 4.0 International License. Read Full License 


\section{Abstract}

Background: Perinatal depression affects $5-15 \%$ of childbearing women. However, literature suggest that Latina women are twice as likely to experience symptoms of depression during pregnancy. Group telehealth-based interventions are a promising approach to increasing access and reducing barriers to mental health services. However, little is known about the experience that childbearing Latinas have with navigating this approach. This paper aimed to explore Latina mothers' perspectives and recommendations in using a telehealth modality to deliver a mindfulness-based cognitive behavioral therapy (MBCBT) group intervention.

Methods: Fourteen pregnant or postpartum women participated in focus groups and individual interviews. Interview topics included perinatal depression, knowledge and content of interventions, and the feasibility and acceptability of the telehealth approach. Data were recorded, transcribed, and analyzed using a grounded theory approach.

Results: Three broad themes emerged; (1) accessibility to telehealth, (2) relational connection through technology, and (3) and technological issues affecting access (i.e., level of familiarity with portal, video quality, etc.). Enhancers promoting participation in the telehealth intervention included reduced scheduling concerns and no need for childcare. Barriers included mistrust of providers' engagement, concerns about privacy, and potential for lack of relational connection through technology.

Conclusion: Specific recommendations were provided to increase participation and effectiveness of telehealth groups among Latinas. Recommendations included conducting an initial assessment of women's familiarity with and access to technology, having a tech-trained individual available to troubleshoot, as well as provider's intentionality in conveying active listening and relational empathy (i.e., using eye contact during telehealth intervention, avoid limited distractions).

Trial Registration: NCT03932760

\section{Introduction}

Perinatal depression is experienced by $5-15 \%$ of childbearing women $(1,2)$. Among Latina women $\square$, rates of perinatal depression have been found to be between $16 \%-56 \%(3,4)$. Despite the over-represented rates of depression, Latinas are less likely to be screened for mental health conditions, less likely be receive an accurate diagnosis, or to receive specialty mental health care when compared non-Latina White population (4-7). Differences in obtaining treatment for the Latino community have been demonstrated, despite positive attitudes towards counseling and use of medication for treating depression held among Latinos (8).

There are multiple intersecting factors potentially influencing the rates of depression and mental health screening within this community, such as immigration status, low socioeconomic levels, and sociocultural stressors when living in the United States $(3,8,9)$. Researchers delineate that specific barriers to 
accessing mental health care among subgroups of Latina women include low mental health literacy, lack of transportation, or lack of childcare (9-11). To an extent, these and other barriers may be mitigated by the use and availability of mental health services using telehealth $(9,12-14)$. A study examining the feasibility of telepsychiatry for low-income Latinx patients found significantly higher visit satisfaction with telepsychiatry than for those receiving traditional face-to-face treatment (13). A recent study examining the use of telehealth during the COVID-19 pandemic among all racial and ethnic groups demonstrated increased use among Latinx participants, suggesting potential as a tool to mitigate access and engagement barriers (15).

Directly related to perinatal depression, a recent systematic review found six studies focused on the use of technology for preventing and treating depression symptoms among childbearing Latina mothers (8). Among the six studies, three were delivering treatment via telephone or smartphone (e.g., text messaging), while the other three were using internet-based interventions. Overall findings demonstrated increase in symptom awareness, mental health literacy, use of resources, and positive attitudes toward the interventions (8). Despite these encouraging findings, there remains a need for studies examining views about telehealth interventions, barriers, and satisfaction for Latina mothers experiencing perinatal depression (9).

Telehealth care delivery may be an accessible option for Latinx women, as this group has high utilization of the internet when compared to other racial-ethnic groups (15). Furthermore, telehealth may be a more accessible and affordable option to care when compared to in-person office visits $(8,14)$. The switch to video conferencing systems during the COVID-19 pandemic has increased utilization and may have shifted perceptions about technology among certain communities (15-17). Additionally, insurance companies increasingly pay for telehealth services, a change which has been accelerated by the COVID19 pandemic. Further studies on optimal modes of telehealth intervention may improve access and participation among the Latinx population, assisting in tailoring telehealth-based therapy for this community.

\section{Study Aim}

This paper presents the findings from a qualitative study exploring Latina mothers' views about telehealth and recommendations regarding a Mindfulness-Based Cognitive Behavioral Therapy (MBCBT) group intervention delivered by videoconference for perinatal depression. We focused on exploring the enhancers and barriers that structurally influence telehealth interventions, as well as the technological considerations during implementation.

\section{MBCBT Intervention}

As part of a randomized controlled trial funded by the National Institute of Nursing Research and the National Institutes of Health at the University of Utah, an MBCBT telehealth group intervention was 
adapted for use among pregnant and postpartum women $(18,19)$. The persistent disparities and reported barriers to receiving perinatal mental health care in the Latina community calls for examination and better understanding of their unique enhancers, barriers, and recommendations for improving quality care, particularly as it relates to group videoconference MBCBT.

\section{Methods}

We conducted two in-person and two virtual focus groups, as well as two virtual individual interviews. Participants self-identified as Latina or Hispanic and were either pregnant or had given birth within the previous 24 months. Eight participants attended in-person focus groups and six participated in virtual focus groups or interviews (total $\mathrm{N}=14$ ).

\section{Data Source and Recruitment}

The recruitment efforts spanned from August 2019 to June 2020 and follow several strategies. We contacted key stakeholders in the community including health workers, primary care physicians, and prenatal educators and asked for advice for best recruiting strategies and culturally sensitive approaches to study engagement. We recruited women at multiple events focused on Latina women's health including community presentations, cultural events, health fairs, and library events. Flyers were shared in libraries, community centers, churches, and prenatal clinics. Social media was also used to promote recruitment and provide information about the study.

The goal was to recruit women to participate in focus groups of 4-6 women, either in-person or via live videoconference. However, individual interviews were conducted to capture the voices of Latina mothers when a lack of participants for a minimum group size occurred. Thus, some participants were asked to join a 1-hour semi-structured interview, while others participated in a 1.5-hour focus group. In both circumstances, a \$20 Amazon or Walmart gift card was provided as compensation for time. The study was approved by the University of Utah Institutional Review Board (IRB \#00113917).

\section{Interview Development and Structure}

The research team sought and received feedback from Latinx community health workers when developing the interview and structuring the focus group meetings. For in-person focus groups, perinatal community health workers suggested creating a comfortable environment for Latina mothers by having snacks, meeting at off-campus locations, having time flexibility, providing transportation, having paper handouts in Spanish and English, and having a bilingual facilitator. These suggestions were integrated into the study implementation. With these in place, the goals for the interview was to (1) explore familiarity, comfort, and barriers with telehealth approaches and to (2) introduce participants to the content of the MBCBT telehealth group intervention (see Table 2). 


\section{Qualitative Data Analysis}

Focus groups were analyzed using a grounded theory analytical approach $(20,21)$. Our coding team sought to discover and construct themes emerging from the data, using a systematic approach to analyze and compare findings. To create a transparent positionality in the way we approached the data, each coder provided a statement about their own identity. The first coder and author of the paper selfidentifies as Afro-Puerto Rican, ciswoman, able-bodied, catholic, and native Spanish-speaking postdoctoral fellow in psychology. The second coder and author of the paper self-identifies as South Asian Indian cis-gender, nonreligious, woman who is a mother and has personal experience with postpartum depression. Lastly, the third coder and author of this paper self-identifies as a White, ciswoman, ablebodied, nonreligious, public health researcher. The coders had continuous process-oriented meetings and memos to examine personal reactions and positionality lenses that informed their coding structure. As a first step, coders approach the data independently, completing an initial open coding procedure. Weekly sessions were held from March 2020 to July 2020 to compare codes, develop axial codes, and identify initial themes. Coders continued refining themes and comparing codes. Coders agreed that saturation was reached by the third focus group, subsequently using the interviews to enhance the understanding of the themes emerging from the focus groups. Following these interviews, a fourth focus group provided new insight regarding the experiences of current participants in the MBCBT program and women who were living in rural Utah.

\section{Results}

The total sample consisted of 14 women who self-identified as either Latina or Hispanic (see Table 1). Participants' ages ranged from 18 to 41 (mean 31.7), with $85 \%$ of the sample indicating they lived in urban areas. The main themes of the focus group and interview data included considerations of technological factors and access to telehealth from a cultural lens (see Figure 1). Subthemes across each area are discussed below. Exemplar quotes and comments from Latina participants related to the specific codes are also included.

\section{Technological Factors}

The following themes were central to practical factors related to the utilization of technology including internet/device access, familiarity with technology, and ease of videoconferencing platform and portal login.

\section{Video quality/Internet access}

Participants noted that not having access to quality internet or having an older device were barriers to entry into telehealth. This appeared to be more of a concern for older women or women who had recently immigrated to the U.S. Two quotes that were illustrative of these factors: 
[I] don't really have the up-and-coming technology that everybody - everybody else has.

...because I ran out of [internet] service and I had no battery left.

\section{Familiarity with technology}

Latina participants indicated that their familiarity with and knowledge of technology would impact their decision to engage in telehealth services. Additionally, participants also discussed age and generational factors impacting use of technology in their day-to-day communications. Participants ranged in only feeling comfortable using phones to text and call families, while others were familiar with videoconferencing platforms such as Zoom, Whatsapp, and Skype. These varying experiences meant that some would feel at ease in engaging in telehealth for perinatal depression while others needed more reassurance and exposure to the platforms.

I had done it [videoconferencing] before, in others, with different ones, then I knew more or less what, what, what I had to do, it was not something new.

Older people maybe having more difficulty login into a system like this one, but it may be possible that younger people is a bit more open to everything that is related to technology.

\section{Ease of videoconferencing platform/portal login}

Participants shared that having a platform and instructions that were accessible and resulted in minimal to no frustrations would promote utilization of telehealth services. They also indicated that it was important to have someone who could troubleshoot quickly when problems arose. Participants indicated that if access to the technology was easy, it often reduced hesitations around engaging with telehealth.

For me it was easy because, you were giving me instructions for me to follow.

It [the portal login] was easy.

I had problems with the password, I don't know why, the good thing is that you were contacting me by text messages, then it was like - it wasn't really a problem because I knew you were there attentive to what I needed in the moment. So, I knew...you were going to fix it and you did it super fast.

\section{Access to Telehealth}

The second broader theme is access to telehealth. Participants described several logistical and relational factors that_facilitated or prevented engagement in telehealth. Specifically, logistical issues such as transportation, scheduling, being busy, and childcare were discussed by the participants. Additional factors that emerged included connections through technology, provider distrust, and privacy concerns. 


\section{Logistics}

Transportation as a barrier to mental health access. Participants expressed that the option to utilize telehealth would remove the barrier of needing transportation to attend doctor's/counselor visits. This was especially true for rural participants. Being able to utilize telehealth for mental health services from home was seen as a benefit.

...so I would have to actually travel $30+$ miles just to get to...a counselor.

... we only have one car.

It is a benefit to take the call from home.

Scheduling \& Being Busy. Scheduling and having time to participate in a telehealth program was a concern for participants who were juggling multiple roles. For some it was challenging to balance time for themselves when navigating work and caring for children or the home. Additionally, some participants felt that as Latinas there was added pressure of working that did not allow for time and participation in mental health services.

...mostly it's the time, isn't it? And sometimes the schedule in which classes are taking place.

...[to] go one needs to have the time.

...everybody from Mexico...[we all] work all the time.

Childcare. Participants also shared that having access to telehealth would be helpful when caretaking for other children. Other participants noted having groups available during the day would be helpful to manage household responsibilities.

...if you still have little ones at home, it would be rather easy [to participate].

... [scheduling] when kids are in school.

\section{Relational Connection through Technology}

Thirdly, the last broad themes relates to aspects of relational connection and how these are influenced by telehealth. Some participants reported that technology enhanced connection with others, while other participants expressed reservation regarding connection through technology. Participants also shared a general distrust in use of technology by providers and their attention to care for these women. The women also shared concerns about having privacy for telehealth in their homes.

\section{Mistrust of Providers when using Technology}


Participants specifically shared concerns that providers may be attending to other things and not giving full attention to them during telehealth visits. They wanted providers to give them full attention and to make eye contact during these visits. The lack of trust within the Latina community may be amplified with the added layer of the screen.

I will be here, and here he [referring to the provider] will be - he will be attending to his affairs.

A little mistrust...hesitation [when having a provider via telehealth].

... the doctor is with other people and the call is supposed to be [with me].

I need you to look me in the eye. If not, l'll just get up and walk away. I'll just.. just forget it. Just forget about it.

\section{Concerns of Privacy}

Participants discussed issues of privacy related to using telehealth at home where they lacked privacy at times. The complexity around undocumented communities being fearful about information getting out intensified the need for privacy and confidentiality. Further, fear of others knowing about their mental health issues which could be heightened within the telehealth context when others are around the home.

I think the fears and barriers would just be them just not feeling sure of where that information - if that information is private, you know, if it's gonna get out.

I mean... it is a benefit to take the call from home, but at the same time I feel that sometimes you [should] be careful in this situation.

I have to be lowering the volume or lowering my voice so that others can't hear. Like I said, I live with three other roommates and I don't want them to be listening when I am there crying or anything like that.

\section{Technology and Relational Connection}

Participants noted that technology at times fostered connection while others worried about losing the connection that is often experienced during in-person groups. The participants noted that having the ability to see other members improved perceived connection with others. Participants noted that glitches in technology would be a hindrance to connection with providers and participants.

The connection is not going to be felt. That is, it will see connection, true, technological, but not the connection that one feels.

...like people would glitch out or we couldn't see one another. 
I was able to see everybody in, like a row - I always got to see everybody...Oh, okay. I made a joke, so I can see everybody laughing.

[I use] technology mainly (to be) connected to other people.

\section{Discussion}

Participants shared perceptions and recommendations regarding technological factors, access to telehealth, and technological considerations. Based on participant feedback, we developed several recommendations that may be helpful in working with Latina mothers within a telehealth approach. Prior to delivering the intervention, participants encouraged the research team to assist in addressing the issues of internet access and access to devices compatible with the intended telehealth platform. Similarly, participants recommended having a tech-trained individual who is available to troubleshoot while simultaneously building trust and rapport as a way to ease participation and comfort in joining a telehealth group. Having sufficient tech support prior and during the delivery of the intervention would support participants who have limited knowledge or experience with technology. Also, women felt that having clear written resources and instructions on how to join the group telehealth session and what to do when unexpected issues arise would be beneficial.

When delivering the intervention, the participants recommended that the facilitator find ways to express interest in women's narratives with strategies such as making direct eye contact with patient through the telehealth webcam, and alerting attendees when they are taking notes or documenting information during sessions. These strategies may prevent participants from feeling ignored, misunderstood, or feeling distant due to multitasking of the facilitator. Women voiced concerns that a lack of personal connection through technology, which could be mitigated by encouraging participants to have their video on and fostering interaction among members. Participants discussed ways in which they have already used technology to connect with others (especially when use of videoconferencing increased during the COVID19 pandemic). Additionally, the participants expressed wanting to discuss concerns related to privacy and joining a group telehealth intervention with unknown members prior to initiation. For instance, participants wanted to brainstormed locations and settings that would be appropriate to join the video call (i.e., car, library, designated office space if needed, etc.; 22). Anecdotally, the participants noted benefits from having access to a bilingual facilitator during the focus groups and interviews - which may ensure participation from women with different levels of comfort in discussing mental health topics in English, Spanish, or a combination of both. To this point, additional research may explore specific cultural and community beliefs about depression influencing engagement in telehealth modalities during the perinatal period.

A limitation of our study is the nature of qualitative research. Given this methodological approach focuses on exploring themes and delving deeper into participant's perception, our small sample of 14 participants limits generalizability. Therefore, our findings are unique to women in this locality. Further, given the heterogeneity of the Latinx community, our findings cannot be generalized across sub- 
communities or intersections of identities (e.g., race, class, education). Our recruitment efforts rendered more participants from urban areas. At times, we encountered challenges in keeping potential participants interested long enough to form a focus group, hence the incorporation of individual interviews in our methodology.

\section{Conclusion}

Themes identified were helpful to tailor the delivery of a telehealth group intervention for Latinas with perinatal and postpartum depression. Telehealth is a promising approach to improving access to mental health care by addressing barriers that prevent Latina mothers from engaging in treatment (23). Despite the challenges and barriers shared by participants, most expressed a desire to engage in mental health treatment and addressing recommendations in this paper would increase accessibility of care.

\section{Abbreviations}

MBCBT: Mindfulness-Based and Cognitive Behavioral Therapy

PD: Perinatal Depression

\section{Declarations}

\section{Ethics approval and consent to participate}

The study was approved by the University of Utah Institutional Review Board (IRB \#00113917). The study involved human subjects and the focus group protocol for involving human data was in accordance with University of Utah's IRB and informed consent was acquired from all participants who were above the age of 18 .

\section{Consent for publication}

Not Applicable

\section{Availability of data and materials}

The datasets generated and/or analysed during the current study are not publicly available due to this being a qualitative study with transcript data that could possibly be identifiable but deididentfied transcripts could be available from the corresponding author on reasonable request.

\section{Methods}


The study design, data collection, and data analyses were carried out in accordance with relevant guidelines and regulations.

\section{Competing interests}

Not Applicable

\section{Funding}

National Institute of Nursing Research

R01NR017620-01A1

\section{Authors' contributions}

Uma Parameswaran and Gwen Latendresse contributed to the design of the study. Uma and Kritzia Merced collected the study group data. Uma, Kritzia, and Susan Dearden analyzed and intrepreted the data and wrote the first draft of the main manuscript. Uma and Kritzia completed the figures and tables in the manuscript. Gwen and Ryoko Pentecost reviewed the data analysis and contributed to the revisions of the manuscript. All authors reviewed and approved the final manuscript.

\section{Acknowledgments}

Not Applicable

\section{References}

1. Lusskin, S.I., Pundiak, T.M. and Habib, S.M. Perinatal depression: hiding in plain sight. The Canadian Journal of Psychiatry, 2007;52(8): 479-488.

2. Gaynes, B.N., Gavin, N., Meltzer-Brody, S., Lohr, K.N., Swinson, T., Gartlehner, G., Brody, S. and Miller, W.C. Perinatal depression: Prevalence, screening accuracy, and screening outcomes: Summary. AHRQ evidence report summaries. 2005

3. Geier ML, Hills N, Gonzales M, Tum K, Finley PR. Detection and treatment rates for perinatal depression in a state Medicaid population. CNS Spectr. 2015;20(1):11-19. doi:10.1017/S1092852914000510

4. Baker-Ericzén MJ, Connelly CD, Hazen AL, Dueñas C, Landsverk JA, Horwitz SM. A collaborative care telemedicine intervention to overcome treatment barriers for Latina women with depression during the perinatal period. Fam Syst Health. 2012;30(3):224-240. doi:10.1037/a0028750 
5. Hahm HC, Cook BL, Ault-Brutus A, Alegría M. Intersection of race-ethnicity and gender in depression care: screening, access, and minimally adequate treatment. Psychiatr Serv. 2015;66(3):258-264. doi:10.1176/appi.ps.201400116

6. Coleman, K.J., Stewart, C., Waitzfelder, B.E., Zeber, J.E., Morales, L.S., Ahmed, A.T., Ahmedani, B.K., Beck, A., Copeland, L.A., Cummings, J.R. and Hunkeler, E.M. Racial-ethnic differences in psychiatric diagnoses and treatment across 11 health care systems in the mental health research network. Psychiatric Services. 2016;67(7):749-757.

7. Alegría M, Canino G, Shrout PE, et al. Prevalence of mental illness in immigrant and non-immigrant U.S. Latino groups. Am J Psychiatry. 2008;165(3):359-369. doi:10.1176/appi.ajp.2007.07040704=

8. Lara-Cinisomo S, Wisner KL, Burns RM, Chaves-Gnecco D. Perinatal depression treatment preferences among Latina mothers. Qual Health Res. 2014;24(2):232-241.

doi:10.1177/1049732313519866

9. Iturralde, E., Hsiao, C.A., Nkemere, L., Kubo, A., Sterling, S.A., Flanagan, T. and Avalos, L.A. Engagement in perinatal depression treatment: a qualitative study of barriers across and within racial/ethnic groups. BMC Pregnancy and Childbirth. 2021;21(1):1-11.

10. Recto, P. and Champion, J.D. Assessment of mental health literacy among perinatal Hispanic adolescents. Issues in Mental Health Nursing. 2017;38(12):1030-1038.

11. Lacsina, J.L., Tang, V.K., Wolitzky-Taylor, K., Brueggmann, D., Jaque, J. and Dossett, E.C. Perceptions of Perinatal Anxiety, Depression, and Mental Health treatment in a Low-Income, Latina Population. Madridge J Womens Health Emancipation, 2017;1(1):22-28.

12. Pflugeisen BM, McCarren C, Poore S, Carlile M, Schroeder R. Virtual Visits: Managing prenatal care with modern technology. MCN Am J Matern Child Nurs. 2016;41(1):24-30. doi:10.1097/NMC.0000000000000199

13. Chong J, Moreno F. Feasibility and acceptability of clinic-based telepsychiatry for low-income Hispanic primary care patients. Telemed J E Health. 2012;18(4):297-304. doi:10.1089/tmj.2011.0126

14. Martinez M, Perle JG. Reaching the Latino Population: A brief conceptual discussion on the use of telehealth to address healthcare disparities for the large and growing population. Jo of Technology in Behavioral Science. 2019;4(3):267-273. doi:10.1007/s41347-019-00088-9

15. Campos-Castillo $C$, Anthony $D$. Racial and ethnic differences in self-reported telehealth use during the COVID-19 pandemic: a secondary analysis of a US survey of internet users from late March. $J \mathrm{Am}$ Med Inform Assoc. 2021;28(1):119-125. doi:10.1093/jamia/ocaa221

16. Monaghesh E, Hajizadeh A. The role of telehealth during COVID-19 outbreak: a systematic review based on current evidence. BMC Public Health. 2020;20(1):1193. doi:10.1186/s12889-020-09301-4

17. Assistant Secretary for Public Affairs (ASPA). Telehealth: Delivering care safely during COVID19. https://www.hhs.gov/coronavirus/telehealth/index.html. Published April 22, 2020. Accessed March 1, 2021.

18. Thompson NJ, Walker ER, Obolensky N, et al. Distance delivery of mindfulness-based cognitive therapy for depression: project UPLIFT. Epilepsy Behav. 2010;19(3):247-254. 
doi:10.1016/j.yebeh.2010.07.031

19. Thompson NJ, Patel AH, Selwa LM, et al. Expanding the efficacy of Project UPLIFT: Distance delivery of mindfulness-based depression prevention to people with epilepsy. J Consult Clin Psychol. 2015;83(2):304-313. doi:10.1037/a0038404

20. Corbin JM, Strauss A. Grounded theory research: Procedures, canons, and evaluative criteria. Qualitative Sociology. 1990;13(1):3-21. doi:10.1007/bf00988593

21. Chun Tie Y, Birks M, Francis K. Grounded theory research: A design framework for novice researchers. SAGE Open Med. 2019;7:2050312118822927. doi:10.1177/2050312118822927

22. George S, Hamilton A, Baker RS. How do low-income urban African Americans and Latinos feel about telemedicine? A Diffusion of Innovation Analysis. Int J Telemed Appl. 2012;2012:715194. doi:10.1155/2012/715194

23. Victorson D, Banas J, Smith J, et al. eSalud: designing and implementing culturally competent ehealth research with latino patient populations. Am J Public Health. 2014;104(12):2259-2265. doi:10.2105/AJPH.2014.302187

\section{Tables}

Table 1

Demographic information about participants 


\begin{tabular}{|c|c|c|}
\hline & Percentage & Missing \\
\hline Ethnicity & & 0 \\
\hline Not Hispanic or Latina & $0(0 \%)$ & \\
\hline Hispanic or Latina & $14(100 \%)$ & \\
\hline Marital Status & & 1 \\
\hline Single & $2(15.4 \%)$ & \\
\hline Married & $8(61.5 \%)$ & \\
\hline Living with partner & $3(23.1 \%)$ & \\
\hline Divorced & $0(0 \%)$ & \\
\hline Widowed & $0(0 \%)$ & \\
\hline Location & & 0 \\
\hline Urban & $12(86 \%)$ & \\
\hline Rural & $2(14 \%)$ & \\
\hline Education Level & & 2 \\
\hline Less than high school & $3(25 \%)$ & \\
\hline High school diploma & $4(33.3 \%)$ & \\
\hline Some college, no degree & $2(16.7 \%)$ & \\
\hline Technical/Certificate Degree & $0(0 \%)$ & \\
\hline College graduate/Bachelor's degree & $2(16.7 \%)$ & \\
\hline Post-graduate degree/Master's & $1(8.3 \%)$ & \\
\hline Doctorate degree & $0(0 \%)$ & \\
\hline Pregnancy Status & & 1 \\
\hline Pregnant & $6(46.2 \%)$ & \\
\hline Postpartum & $7(53.8 \%)$ & \\
\hline Income & & 3 \\
\hline Less than $\$ 10,000$ & $2(18.2 \%)$ & \\
\hline$\$ 10,000-\$ 29.999$ & $4(36.4 \%)$ & \\
\hline$\$ 30,000-\$ 49,000$ & $4(36.4 \%)$ & \\
\hline$\$ 50,000-\$ 69,999$ & $1(9.1 \%)$ & \\
\hline$\$ 70,000-\$ 99,999$ & $0(0 \%)$ & \\
\hline
\end{tabular}

Page 14/ 16 


\begin{tabular}{|c|c|}
\hline Greater than $\$ 100,000$ & $0(0 \%)$ \\
\hline Language Preference & 0 \\
\hline English & $5(36 \%)$ \\
\hline Spanish & $9(64 \%)$ \\
\hline
\end{tabular}

Table 2

Interview Structure

1. Familiarity and comfort with telehealth interventions
- Prior experience with telehealth interventions

- Feedback about current platform and comfort

- Potential barriers and enhancers in using modality

2. Introduce to the MBCBT telehealth group intervention content
- Language component and facilitators preference

- Treatment structure and therapeutic concepts

\section{Figures}




\section{Connection through Technology}

- Mistrust of providers when using technology

- Concerns of privacy

- Technology \& relational connection

\section{Access to Telehealth}

- Logistics

- Scheduling \& being busy

- Childcare
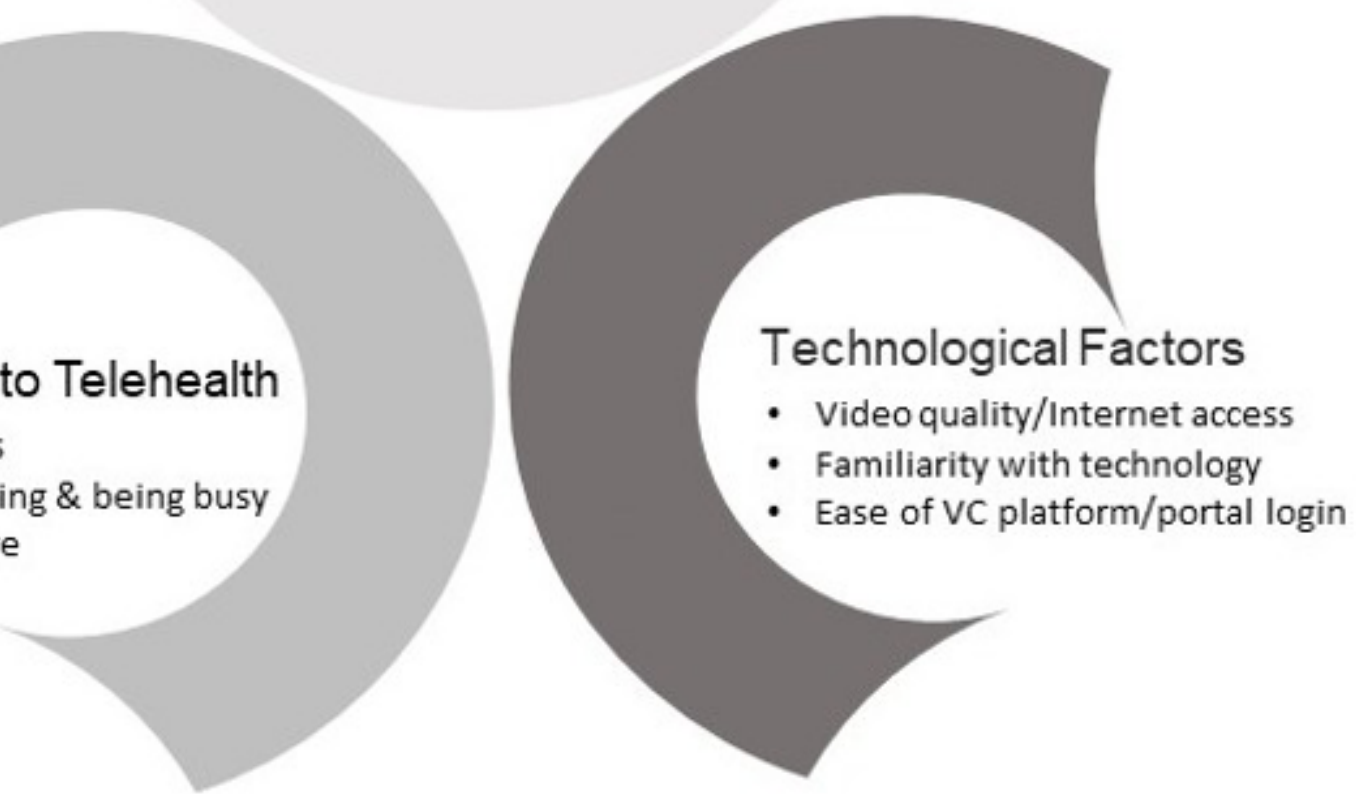

\section{Figure 1}

Subthemes across each area are discussed below. Exemplar quotes and comments from Latina participants related to the specific codes are also included. 